\title{
Breaking of axial symmetry in excited nuclei as identified in experimental data
}

\author{
Arnd R. Junghans ${ }^{1, a}$, Eckart Grosse ${ }^{2, b}$, and Ralph Massarczyk ${ }^{1,2}$ \\ 1 Helmholtz-Zentrum Dresden-Rossendorf, Bautzner Landstrasse 400, 01328 Dresden, Germany \\ 2 Technische Universität Dresden, Institut für Kern- und Teilchenphysik, Zellerscher Weg 19, 01069 Dresden, Germany
}

\begin{abstract}
A phenomenological prediction for radiative neutron capture is presented and compared to recent compilations of Maxwellian averaged cross sections and average radiative widths. Photon strength functions and nuclear level densities near the neutron separation energy are extracted from data without the assumption of axial symmetry - at variance to common usage. A satisfactory description is reached with a small number of global parameters when theoretical predictions on triaxiality (from constrained HFB calculations with the Gogny D1S interaction) are inserted into conventional calculations of radiative neutron capture. The photon strength is parametrized using the sum of three Lorentzians (TLO) in accordance to the dipole sum rule. The positions and widths are accounted for by the droplet model with surface dissipation without locally adjusted parameters. Level densities are influenced strongly by the significant collective enhancement based on the breaking of axial symmetry. With the less stringent requirement of invariance against rotation by $180^{\circ}$ a global set of parameters which allows to describe the photon strength function and the level densities in the nuclear mass range from mass number $50<\mathrm{A}<250$ is found.
\end{abstract}

\section{Introduction}

A triaxial shape for heavy nuclei should provide a more general basis for the description of nuclear structure than the assumption of axial symmetry, which probably originates from atomic hyperfine structure observations [1], which are usually made on unpolarized probes and thus are insensitive to broken axiality. This does not exclude the possibility that certain properties as observed in certain nuclei may be reproduced well by a theoretical model based on axial or even spherical symmetry. Multiple Coulomb excitation experiments and the shape of the Giant Dipole Resonance are rather sensitive probes to the triaxial shape of nuclei. Non axial symmetric shapes have been found in many transitional nuclei studied in multiple Coulomb excitation [2-4].

A recent constrained HFB calculation using the Gogny D1S interaction by Delaroche et al. [5] indicates broken axial symmetry for a large number of nuclei across the table of isotopes. A clustering of values around $Q_{0} \approx 200 \mathrm{fm}^{2}$ and $\cos (3 \gamma) \approx 0.2$ is a result of this calculation where in some cases the values including the predicted standard deviation do not include $\gamma=0^{\circ}$ i.e., $\cos (3 \gamma)=1$. In this paper, the isovector Giant Dipole Resonance (GDR) shape and Maxwellian averaged cross sections will be used as sensitive probes on axial symmetry breaking in nuclei.

The GDR will be parametrized without the a priori assumption of axial symmetry using three Lorentzian terms [6]. The nuclear level density will be described on the same grounds leading to collective enhancement [7].

\footnotetext{
a e-mail: a.junghans@hzdr.de

b e-mail: e.grosse@tu-dresden.de
}

A purely phenomenological parametrization of minor strengths on the low energy tail of the GDR is presented based on most of the available experimental data $[8,9]$. With these quantities averaged neutron capture cross sections can be calculated and are compared with experimental Maxwellian averaged cross sections [10,11].

\section{Triple Lorentzian parameterisation of the GDR}

A global description of the GDR in all heavy nuclei without fitting Lorentzian parameters to individual photonuclear data can be reached using the triple Lorentzian parametrization TLO [6].

The centroid energies $E_{k}$ are determined with

$$
E_{k}=\frac{R_{0}}{R_{k}} E_{0}
$$

where $E_{0}$ is calculated from the Droplet Model [12], axes ratios, charge radii and deformation parameters $\beta, \gamma$ [5]. As the deformation parameters close to magic numbers are overestimated by the calculation the value of the quadrupole deformation $\beta$ is reduced for nuclides ten or less nucleons away from the shell closure by

$$
\beta \rightarrow \beta\left(0.4+\frac{N-N_{\text {shell }}}{20}\right)
$$

The GDR width is based on a hydrodynamical model [13]

$$
\Gamma_{k}=0.045 E_{k}^{1.6}
$$

The TRK sum rule is used for normalization of the GDR region [14]. 


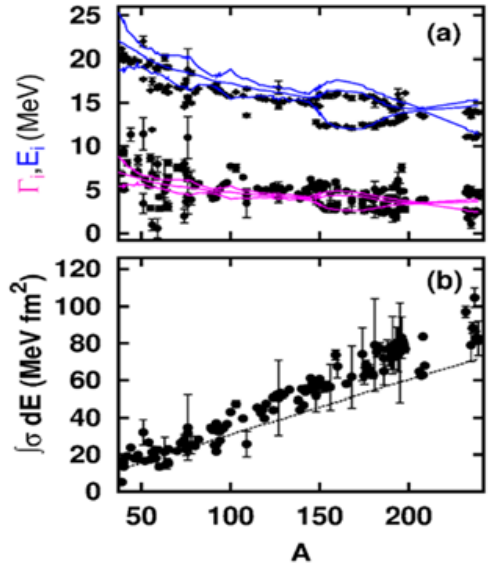

Figure 1. The data points in panel (a) show the energies (top) and widths (bottom) vs. mass number resulting from fits to GDR photonuclear data in heavy nuclei taken from Ref. [15]. The fits are based on one or two Lorentzians and two points per nucleus are shown, if a two Lorentzian fit led to a better description oft he data. Our calculations (TLO) as described in Sect. 2 are depicted as full lines for the three components of the GDR; (blue for $E_{i}$ and magenta for $\Gamma_{i}$ ). In panel (b) the resulting GDR-integrals as obtained by the Lorentzian fits from Ref. [16] are depicted in comparison to the TRK sum rule (dotted line), which is surpassed considerably in most cases, whereas TLO obeys it by definition.

The photonuclear absorption cross section is given by

$$
\sigma_{\mathrm{abs}}^{\mathrm{E} 1}\left(E_{\gamma}\right) \cong 4 \pi \frac{\alpha \hbar^{2}}{m_{N}} \mathrm{fm}^{2} \frac{Z N}{3 A} \sum_{\mathrm{k}=1}^{3} \frac{E_{\gamma}^{2} \Gamma_{k}}{\left(E_{k}^{2}-E_{\gamma}^{2}\right)^{2}+E_{\gamma}^{2} \Gamma_{k}^{2}}
$$

Figure 1 shows the GDR centroid energies and widths for the global TLO parametrization in comparison with local one or two Lorentzian fits. Strong variations in the local fits occur due depending on the use of one or two Lorentzians. As a consequence the TRK sum rule is overfullfilled using this approach and even neighbouring nuclei can differ significantly in the integrated cross sections.

The TLO parametrization is compared with photoneutron cross sections for Sm-isotopes measured using positron-annihilation in flight [17]. see Fig. 2. The splitting of the GDR in three components of equal area allows a good description of the photonuclear cross sections. The apparent increase of the spreading width is related to the split of the centroid energies $E_{i}$ and not to an increase of the spreading width $\Gamma$. If the data in the upper panel were fitted using a single Lorentzian, the resulting width would be much larger than given by Eq. (3) and indicate an overshoot of the TRK sum rule. The effect of instantaneous shape sampling based on the variances of the deformation parameters is shown to be small. The experimental data have been renormalized with a factor 0.9 according to Refs. [18, 19].

\subsection{Empirical parametrization of minor strength}

The height of the low energy tail is nearly proportional to the GDR width and it depends weakly on its deformation induced splitting. A consequence hereof is a nearly full independence of the strength on any free parameter. Photon scattering experiments observed in this energy range

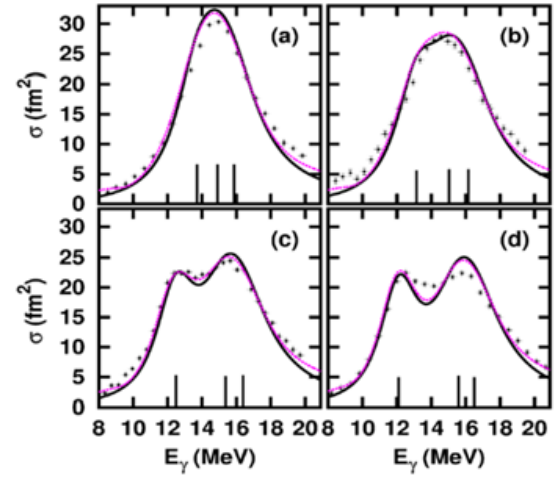

Figure 2. Photo-neutron production cross section ${ }^{148,150,152,154} \mathrm{Sm}$ data points, Ref. [17] (a) to (d) in comparison to the TLO sum of three Lorentzians (full curves) with $E_{i}$ indicated as black bars. The dashed (magenta) curves indicate the effect of shape sampling [20]. The data were renormalized by a factor 0.9 and the calculations were folded with a Gaussian to simulate the experimental beam spread of $\sigma=0.3 \mathrm{MeV}$.

mainly narrow peaks, which were interpreted as strength apparently of other character than isovector electric dipole [8,9,21-23]. Even if it has minor importance for total photon absorption and the sum rules, it may influence the decay of the compound nucleus and consequently also the cross sections of capture reactions.

Here at least their average electromagnetic strength has to be regarded. For an assessment of the agreement between TLO and experimental data the strength eventually adding to the GDR tail has to be characterized; if it is not of electric isovector kind it has to be described separately from the GDR, as described by the TLO parametrization. As a first step of such a characterization we have derived phenomenological expressions for these 'minor' modes in their $A$-dependence and compare them to data, which are partly obtained at the ELBE facility at Dresden and partly derived from published work. We list the obtained parameters in Table 1 and consider this as a basis for eventual theoretical work addressing especially strength issues. The parameters are to be used with a Gaussian function for the corresponding strength distribution

$$
\begin{aligned}
& f_{\lambda}\left(E_{\gamma}\right) \\
& \quad=\frac{1}{(\pi \hbar c)^{2} g_{e f f} E_{\lambda}^{2 \lambda-1}} \frac{I_{c}}{\sqrt{2 \pi} \sigma_{c}} \exp \left(-\frac{\left(E_{\gamma}-E_{c}\right)^{2}}{2 \sigma_{c}^{2}}\right)
\end{aligned}
$$

The parameters were selected to somewhat overpredict the experimental data after the TLO integral over the energy interval of the distribution is subtracted. The use of a Gaussian function suppresses unobservable strength in long tails. In Fig. 3 the TLO strength with and without minor strength according to Table 1 is shown in comparison with data from ${ }^{197} \mathrm{Au}$. Figure 3 shows that the single lorentzian fit (SLO) can lead to an over-estimation of the tail.

\section{Averaged neutron capture cross sections}

The radiative capture of neutrons in the $\mathrm{keV}$ to $\mathrm{MeV}$ range in the range of unresolved resonances can be described 
Table 1. Parameters of an upper limit for three minor electric and three magnetic dipole modes to the dipole strength function to be calculated with a Gaussian, see Eq. (5). A very similar shape near the peak is reached for a Gaussian with standard deviation $\sigma$, which is larger by a factor 2.5 as compared to the width $\Gamma$ of a Lorentzian. Three GQR modes are also listed, which are seen in photon absorption as well; they are proportional to the square of the nucleus' charge radius $R_{p}(\mathrm{rms})$.

\begin{tabular}{ccccc}
\hline Component & Multipolarity & $E_{c}(\mathrm{MeV})$ & $I_{c}\left(\mathrm{fm}^{2} \mathrm{MeV}\right)$ & $\sigma_{c}(\mathrm{MeV})$ \\
\hline Low $E_{x}$ pygmy mode & $\mathrm{E} 1$ & $0.43 E_{0}$ & $7 \frac{Z(N-Z)}{A}$ & 0.6 \\
High $E_{x}$ pygmy mode & $\mathrm{E} 1$ & $0.55 E_{0}$ & $13 \frac{Z(N-Z)}{A}$ & 0.5 \\
$0^{+} \leftrightarrow\left(2^{+} \times 3^{-}\right) 1_{1^{-}}$ & $\mathrm{E} 1$ & $\frac{140}{N}\left(1+\frac{107}{Z}\right)$ & $0.006 Z A \beta$ & 0.6 \\
Orbital $($ scissors) mode & $\mathrm{M} 1$ & $0.21 E_{0}$ & $0.033 Z A \beta$ & 0.4 \\
Isoscalar spin-flip & $\mathrm{M} 1$ & $42 A^{-1 / 3}$ & 17 & 0.8 \\
Isovector spin-flip & $\mathrm{M} 1$ & $47 A^{-1 / 3}$ & 27 & 1.3 \\
Low $E_{x}$ quadrupole mode & $\mathrm{E} 2$ & $19 A^{-1 / 3}$ & $0.1 \frac{\alpha_{e}\left(\pi R_{c} E_{\gamma}\right)^{2} Z^{2}}{3 A m_{p} c^{2}}$ & 1.0 \\
ISGQR & $\mathrm{E} 2$ & $63 A^{-1 / 3}$ & $2 \frac{\alpha_{e}\left(\pi R_{c} E_{\gamma}\right)^{2} Z^{2}}{3 A m_{p} c^{2}}$ & 1.0 \\
IVGQR & & & $\frac{\alpha_{e}\left(\pi R_{c} E_{\gamma}\right)^{2} Z^{2}}{3 A m_{p} c^{2}}$ & 1.8 \\
\hline
\end{tabular}

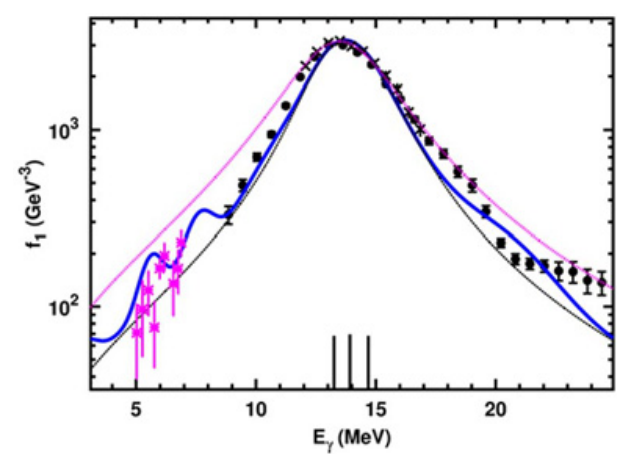

Figure 3. TLO parametrization without minor strength (full black line), minor strength included (thick blue line) and a SLO fit from Ref. [15] compared with experimental data for ${ }^{197} \mathrm{Au}$ : The $(\gamma, \mathrm{n})$ data $[18,24]$ (black data points) are scaled with 0.9 photon scattering data [25] are shown as magenta data symbols.

by statistical model calculations. Their reliability depends not only on the proper description of the input channel, but more strongly on the details determining the decay of the intermediately formed compound nucleus. Here the strength of its electromagnetic decay is of importance as well as the available phase space in the final nucleus, i.e., the density of levels reached by the first photon emitted. To derive a parameterization which is global and thus expected to be applicable also away from stable nuclei, it should rely on concepts valid generally and directly account for effects of nuclear shells and shapes. As is well known, the variation of nuclear quadrupole moments over the nuclide chart is very significant. It thus is indicated to also investigate the influence of shape symmetries on nuclear level densities.

\subsection{Level densities in nuclei without axial symmetry}

The effect of triaxiality on the nuclear level density has been described in an analytical approach on an absolute scale for the small $J$ limit as is applicable for spin-0 target nuclei and low energy neutron capture [11]. The level density is described using a constant temperature formula with pairing dominated properties below a critical temperature and a backshifted Fermi-Gas above. This is in analogy to the model by Gilbert and Cameron [26]. The level density parameters, backshift and phase-transition energy $\tilde{a}, \mathrm{E}_{\mathrm{bs}}, \mathrm{E}_{\mathrm{pt}}$ were not fitted to experimental data. The state density is described by

$$
\omega_{q p}\left(E_{x}\right)=\omega_{q p}(0) \exp \left(\frac{E_{x}}{T_{c t}}\right)
$$

below the phase transition energy and above by

$$
\omega_{q p}\left(E_{x}\right)=\frac{\sqrt{\pi} \exp \left(2 \sqrt{\tilde{a}\left(E_{x}-E_{b s}\right)}\right)}{12 \tilde{a}^{1 / 4}\left(E_{x}-E_{b s}\right)^{5 / 4}}
$$

The phase transition energy is given by $E_{p t}=\tilde{a} t_{p t}^{2}+E_{b s}$ with $t_{p t}=0.567 \Delta_{0}$ using a constant pairing gap of $\Delta_{0}=$ $12 \mathrm{MeV}^{-1 / 2}$. The Fermi-gas level density parameter is described by the nuclear matter value adding a surface correction from a fit to neutron resonance data

$$
\tilde{a}=\frac{\pi 2 A}{4 \varepsilon_{F}}+0.06 \mathrm{MeV}^{-1} A^{2 / 3}
$$

The backshift parameter is determined by

$$
E_{b s}=\frac{3}{8} \frac{A}{\varepsilon_{F}} \Delta_{0}^{2}-\delta E(Z, A)
$$

using the shell correction $\delta E(Z, A)$ from Ref. [27]. The level density parameters are discussed in detail in Ref. [11].

The level density is approximated in the case of small $J$ including the collective enhancement described by Bjornholm et al. [7].

$$
\rho\left(E_{x}, J\right) \stackrel{\text { small } J}{\rightarrow} \frac{2 J+1}{4} \omega_{q p}\left(E_{x}\right)
$$

This approach corresponds to a triaxial nuclear shape having only R-symmetry. The average neutron capture 


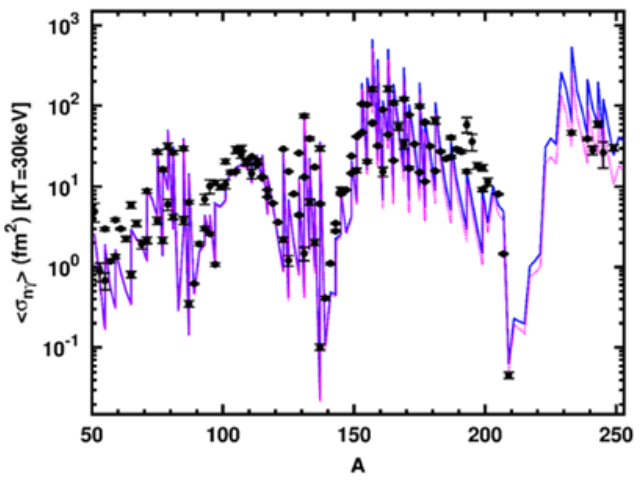

Figure 4. Maxwellian averages of experimental cross sections $[10,28]$ for radiative neutron capture into even nuclei with $\mathrm{J}=0$ and $50<\mathrm{A}<250$ shown as black data symbols for $\mathrm{kT}=30 \mathrm{keV}$. They are plotted vs. $A_{c n}$ in comparison to calculations based on Eq. (11) with TLO (dotted line in magenta) and including the minor components listed in Table I (full blue line). The level density predictions include a damping of the shell effect.

cross cross section can be calculated above the keV-range using

$$
\begin{aligned}
& \sigma_{n \gamma} \cong \\
& 2 \pi^{2} \hbar^{2} \sum_{J_{b}} g^{\prime} \int_{0}^{E_{r}} f_{1}\left(E_{\gamma}\right) E_{\gamma}^{3} \cdot \rho\left(E_{b}, J_{b}\right) d E_{\gamma}
\end{aligned}
$$

Using Eq. (11) averaged over a Maxwellian energy spectrum experimental Maxwellian averaged cross sections can be calculated. A reasonable agreement with experimental data is shown in Fig. 4. The influence of the minor strengths in these nuclei is rather small.

\section{Conclusions}

By allowing general triaxial shapes as indicated in a recent constrained HFB calculation using the Gogny D1S interaction [5] for ground states and low excited states, allows to give a global description of the GDR photonuclear cross sections. Applying the collective enhancement to the nuclear level density for triaxial shapes [7] allows us to describe the level density around the neutron binding energy without local fits of the level density parameters. A reasonable description of Maxwellian averaged neutron capture cross sections can be reached for spin- 0 target nuclei. These findings indicate that the restriction to axially symmetric shapes for nuclei might not be justified and axial symmetry is broken also at higher excitation energies.

This work was supported by the European Commission within the Seventh Framework Programme through Fission-2013CHANDA (project no. 605203).

\section{References}

[1] H. Schiller, U.Th. Schmidt, Zeits. f. Phys. 94, 457 (1935), id., 95, 265 (1935); id., 98, 430 (1936)

[2] C.Y. Wu and D. Cline, Phys. Rev. C 54, 2356 (1996)

[3] W. Andrejtscheff and P. Petkov, Phys. Rev. C 48, 2531 (1993); id., Phys. Lett. B 329, 1 (1994)

[4] J. Srebnry et al., Int. J. of Mod. Phys. E 20, 422 (2011) and refs. quoted therein

[5] J.-P. Delaroche et al., Phys. Rev. C 81, 014303 (2010)

[6] A.R. Junghans et al., Phys. Lett. B 670, 200 (2008)

[7] S. Bjornholm, A. Bohr and B. Mottelson, Physics and Chemistry of Fission, Rochester, IAEASTI/PUB/347 (1974) 367; dto., IAEA-SM-174 205

[8] K. Heyde, P. von Neumann-Cosel, A. Richter, Rev. Mod. Phys. 82, 2365 (2010)

[9] E. Grosse and A.R. Junghans, Landolt-Börnstein, New Series (2012) I/25D, 4

[10] I. Dillmann et al., Phys. Rev. C 81, 015801 (2010); id., AIP Conf. Proc. 819, 123; http: //www . kadonis .org

[11] E. Grosse, A.R. Junghans and R. Massarczyk, Phys. Lett. B 739, 1 (2014)

[12] W.D. Myers et al., Phys. Rev. C 15, 2032 (1977)

[13] B. Bush and Y. Alhassid, Nucl. Phys. A 531, 27 (1991)

[14] M. Gell-Mann et al., Phys. Rev. 95, 1612 (1954)

[15] V.A. Plujko et al., At. Data and Nucl. Data Tables 97 567 (2011); http: //www-nds . iaea.org/RIPL-3/ gamma

[16] R. Capote et al., Nucl. Data Sheets 110, 3107 (2009); id., http: //www-nds . iaea . org/RIPL-3

[17] P. Carlos et al., Nucl. Phys. A 225, 171 (1974)

[18] B.L. Berman et al., Phys. Rev. C 36, 1286 (1987)

[19] C. Nair et al., Phys. Rev. C 81, 055806 (2010)

[20] S.Q. Zhang et al., Phys. Rev. C 80, 021307 (2009)

[21] G.A. Bartholomew et al., Adv. Nucl. Phys. 7, 229 (1973)

[22] U. Kneissl et al., J. Phys. G 32, R217 (2006)

[23] D. Savran et al., Prog. Part. Nucl. Phys. 70, 210 (2013); id., Phys. Rev. Lett. 100, 232501 (2008)

[24] A. Lepretre et al., Nucl. Phys. A 219, 39 (1974)

[25] P. Axel et al., Phys. Rev. C 2, 689 (1970)

[26] A. Gilbert and A.G.W. Cameron, Can. Journ. of Physics 43, 1446 (1965)

[27] W.D. Myers, W.J.Swiatecki, Ark. Fizik 36, 343 (1967)

[28] B. Pritychenko et al., At. Data and Nucl. Data Tables 96, 645 (2010) 